\title{
Tim-1 alleviates lupus nephritis-induced podocyte injury via regulating autophagy
}

\author{
YUNXIA YU, CAIXIA ZHU, NAN YU, LIJUAN YANG
}

Department of Rheumatology, the General Hospital of Ningxia Medical University, Yinchuan, Ningxia Province, China

\begin{abstract}
Introduction: Lupus nephritis (LN) is a complication of systemic lupus erythematosus (SLE) which seriously threatens the health of people. Tim-1 is known to be associated with the pathogenesis of SLE. However, the role of Tim-1 in LN is still unclear.

Aim of the study: To explore the expression and the potential regulatory molecular mechanism of Tim-1 in LN-induced podocyte injury.

Material and methods: An in vivo model of LN was established to detect the expression of Tim-1, inflammatory cytokines and autophagy-related proteins. Podocytes were treated with immunoglobulin $G$ $(\mathrm{IgG})$ to establish the LN in vitro model and then treated with an autophagy inhibitor. RT-qPCR and western blot were performed to investigate the effect of Tim-1 on inflammatory responses as well as autophagy in podocytes. The function of Tim-1 in IgG-induced podocytes was detected by CCK-8 and flow cytometry, respectively.

Results: Tim-1, L3BII/L3BI ratio and inflammatory cytokines were upregulated in LN mice. Tim-1 notably inhibited IgG-induced inflammatory responses in podocytes via reducing tumor necrosis factor $\alpha$ (TNF- $\alpha)$, interleukin (IL)-6 and IL-1 $\beta$ expression, and it could protect podocytes against $L N$-induced injury via inducing autophagy. Meanwhile, Tim-1 significantly promoted the proliferation of IgG-induced podocytes via inhibiting apoptosis. The autophagy inhibitor reversed the effect of Tim-1 on inflammatory cytokines and autophagy-related proteins in $\lg G$-treated podocytes.

Conclusions: Tim-1 protects podocytes against LN-induced injury via mediating autophagy, which might serve as a new target for the treatment of $L N$.
\end{abstract}

Key words: lupus nephritis (LN), lupus nephritis, Tim-1, podocytes, autophagy.

(Cent Eur J Immunol 2021; 46 (3): 305-313)

\section{Introduction}

Systemic lupus erythematosus (SLE) is a spectrum of autoimmune disease in which defects can occur at multiple points of the immune cascade [1]. At present, SLE is recognized with 2019 European League Against Rheumatism/ American College of Rheumatology (EULAR/ACE) criteria [2]. In addition, SLE can lead to a striking heterogeneity of clinical presentations [3]. The central pathology is the recognition of, and reaction to, self-antigens by the immune system, leading to type I interferon signaling and the production of pathogenic auto-antibodies, which mediate much of the disease [2, 4]. Lupus nephritis (LN) is one of the most serious manifestations of SLE and is a crucial predictor of poor outcome [5], which is classified with revised International Society of Nephrology criteria [4]. Occurrence and progression of $\mathrm{LN}$ are the end result of complex interactions between regulation of immune responses and the pathological process involving renal resident cells $[6,7]$. Moreover, the progression of $\mathrm{LN}$ may lead to the occurrence of renal failure [8]. Nowadays, the main treatment of LN includes glucocorticoid and immunosuppressive therapy [9]. However, the therapeutic effect is still not ideal. Thereby, it is necessary to find new strategies for the treatment of $\mathrm{LN}$.

$\mathrm{T}$ cell immunoglobulin and mucin 1 (Tim-1) is an important gene that mediates T-helper cell development [10]. Tim- 1 is expressed in CD4 $4^{+} \mathrm{T}$ cells and starts transcription at the initial stage of antigen stimulation, which provides a costimulatory signal for $\mathrm{T}$ cell activation, participates in $\mathrm{T}$ cell proliferation and differentiation, and inhibits the occurrence of peripheral tolerance [11-13]. These findings suggest that Tim-1 is a key gene that can regulate T cells and is likely to be an immune marker. Meanwhile, Tim-1 has been proved to inhibit the progression of $\mathrm{LN}[14,15]$. However, the regulatory role of Tim-1 in LN is largely unknown.

Autophagy stands for an ability of cells to prevent their own death [16], which could also protect cells against apoptosis [17]. In addition, some reports have proved that autophagy can be an important mediator in the immune system $[18,19]$. Meanwhile, autophagy is closely associated with progression

Correspondence: Yunxia Yu, the General Hospital of Ningxia Medical University, No. 840, Shengli South St., Xingqing District, Yinchuan, Ningxia Province, China, phone: +86 13995181603, e-mail: fsyyx94@ sina.com Submitted: 10.12.2020; Accepted: 13.04.2021 
of SLE/LN. For instance, autophagy may contribute to the progression of SLE [20]. Yang et al. reported that autophagy can promote the occurrence of LN [21]. Thus, autophagy plays a key role in LN. It has been previously reported that Tim-1 is closely related with autophagy. For example, Tim-1 may participate in the activation of Dengue virus-induced autophagy [22]. Tim-1-mediated phagocytosis can link ATG5-/ULK1-dependent clearance of apoptotic cells to antigen presentation [23]. However, the association between Tim-1 and autophagy in LN remains unclear.

In the current study, we sought to investigate the function of Tim-1 in LN in vitro. We investigated the function of Tim-1 in LN in vitro as well as the correlation between Tim-1 and autophagy in LN. We hope our research will provide a new strategy for the treatment of $\mathrm{LN}$, as well as a new hope for patients with LN.

\section{Material and methods}

\section{Cell culture and treatment}

Human immortalized podocytes were obtained from American Type Culture Collection and cultured in RPMI1640 medium (Thermo Fisher Scientific, Waltham, MA, USA), supplemented with $10 \%$ fetal bovine serum (FBS; Thermo Fisher Scientific) and $2 \mathrm{mM}$ glutamine (Sigma, St. Louis, MA, USA) at $37^{\circ} \mathrm{C}$. Podocytes were treated with immunoglobulin G (IgG; Sigma) for $24 \mathrm{~h}$ to establish an in vitro model of $\mathrm{LN}$. To confirm the correlation between Tim-1 and autophagy in LN, podocytes were treated with an autophagy inhibitor (3-methyladenine and bafilomycin A1, Sigma).

\section{Cell transfection}

Tim-1 overexpression (pcDNA-3.1-Tim-1) and empty vector (pcDNA3.1) were purchased from Genepharma (Shanghai, China), and were transfected into podocytes using Lipofectamine 2000 (Thermo Fisher Scientific) according to the manufacturer's instructions.

\section{In vivo study}

Ten female MRL ${ }^{\text {Ipr/pr }}$ lupus prone mice and 5 control female C57BL mice were acquired from Nanjing Medical University. C57BL mice served as control mice. In addition, MRL ${ }^{\mathrm{lp} / / \mathrm{pr}}$ lupus prone mice were randomly divided into 2 groups: MRL ${ }^{\text {pr/pr }}$ and LN. Mice in the control and MRL ${ }^{1 p r / p r}$ groups were anesthetized by an intraperitoneal injection of $2 \%$ pentobarbital sodium at a dose of $45 \mathrm{mg} / \mathrm{kg}$ body weight at the age of 3 months, while mice in the LN group were injected at the age of 6 months. Kidney tissues were collected from mice and harvested. All the procedures were approved by the Institutional Animal Care and Use Committee of the local hospital.

\section{Cell Counting Kit-8 (CCK-8) assay}

The CCK-8 assay was used to assess cell proliferation according to the manufacturer's protocol. Podocytes were plated $\left(5 \times 10^{3}\right.$ cells/well) into $96-$ well plates and treated for 24,48 or $72 \mathrm{~h}$ at $37^{\circ} \mathrm{C}$ as follows: Tim-1, IgG or Tim- $1+$ IgG. Subsequently, podocytes were incubated with $10 \mu \mathrm{l}$ of CCK-8 reagents (Beyotime, Shanghai, China) for $2 \mathrm{~h}$ at $37^{\circ} \mathrm{C}$. The absorbance of each well was measured at a wavelength of $450 \mathrm{~nm}$ using a microplate reader (Thermo Fisher Scientific).

\section{Cell apoptosis analysis}

Podocytes were seeded in a 6-well plate $\left(5 \times 10^{4}\right.$ per well). Cells were centrifuged at $956 \mathrm{~g}$ for $5 \mathrm{~min}$ and the residue was resuspended with $100 \mu \mathrm{l}$ of binding buffer. Subsequently, $5 \mu \mathrm{l}$ of Annexin V-FITC and $5 \mu \mathrm{l}$ of propidium iodide were added to the cells for $15 \mathrm{~min}$ at $4^{\circ} \mathrm{C}$. Apoptotic cells were analyzed by flow cytometry using a flow cytometer (Becton, Dickinson and Company, USA).

\section{ELISA}

The levels of interleukin (IL)-1 $\beta$, IL-6, and tumor necrosis factor $\alpha$ (TNF- $\alpha$ ) in supernatants of podocytes or serum of mice were detected using an ELISA kit (Multisciences (Lianke) Biotech Co., Ltd, Hangzhou, China) according to the manufacturer's instructions.

\section{Reverse transcription-quantitative PCR}

Total RNA was extracted from cell lines or tissues using TRIzol reagent (Thermo Fisher Scientific) according to the manufacturer's protocol. Total RNA was reverse transcribed into cDNA using the PrimeScript RT reagent kit (Takara, Tokyo, Japan). Subsequently, reverse transcription-quantitative polymerase chain reaction (RT-qPCR) was performed using the SYBR premix Ex Taq II kit (Takara, Tokyo, Japan). Real-time qPCRs were performed in triplicate under the following protocol: 2 minutes at $94^{\circ} \mathrm{C}$, followed by 35 cycles $(30 \mathrm{~s}$ at $94^{\circ} \mathrm{C}$ and $45 \mathrm{~s}$ at $55^{\circ} \mathrm{C}$ ). The following primer pairs were used for RT-qPCR: Tim-1 forward, 5'-GGAAGTGGTGGCTATGAGTCAG-3' and reverse, 5'-TGTCAATTTGAAACTTAAAAAGCAG-3'; IL-6 forward, 5'-GGAAGTGGTGGCTATGAGTCAG-3' and reverse, 5'-TGTCAATTTGAAACTTAAAAAGCAG-3'; IL-1 $\beta$ forward, 5'-GGAAGTGGTGGCTATGAGTCAG-3' and reverse, 5'-TGTCAATTTGAAACTTAAAAAGCAG-3'; TNF- $\alpha$ forward, 5'-GGAAGTGGTGGCTATGAGTCAG-3' and reverse, 5'-TGTCAATTTGAAACTTAAAAAGCAG-3'; $\beta$-actin forward, 5'-GTCCACCGCAAATGCTTCTA-3' and reverse, 5'-TGCTGTCACCTTCACCGTTC-3'. The gene levels were quantified using the $2^{-\Delta \Delta c t}$ method and $\beta$-actin was used as an internal reference.

\section{Western blotting}

Total protein was extracted from cell lines or tissues using RIPA lysis buffer (Beyotime, Shanghai, China). Total protein was quantified using the bicinchoninic acid protein kit (Thermo Fisher Scientific). Protein (40 $\mu$ g per lane) was separated with $10 \%$ SDS-PAGE and transferred onto PVDF membranes 
(Thermo Fisher Scientific). Subsequently, the membranes were blocked with 5\% skimmed milk in TBST for $1 \mathrm{~h}$ at room temperature. The membranes were incubated overnight at $4{ }^{\circ} \mathrm{C}$ with primary antibodies against: IL-6 (Abcam; ab239007, 1 : 1000), IL-1 $\beta$ (Abcam; ab118973, $1: 1000)$, TNF- $\alpha$ (Abcam; ab219620, 1 : 1000), LC3 (Abcam; ab136668, 1 : 1000), p62 (Abcam; ab109330, 1 : 1000), Tim-1 (Abcam; ab5666, $1:$ 1000) and GAPDH (Abcam; ab179467, $1: 1000)$. Then, the membranes were incubated with HRP-conjugated secondary antibodies (Abcam; ab7356, $1: 5000$ ) for $1 \mathrm{~h}$. Protein bands were visualized using the ECL kit (Thermo Fisher Scientific). $\beta$-actin was used as the loading control. IPP 6.0 (Image-Pro Plus 6.0) was used for the densitometry analysis.

\section{Statistical analysis}

Data are presented as the mean \pm standard deviation. Comparisons between two groups were analyzed by Student's $t$-test. Comparisons among multiple groups were analyzed using oneway ANOVA followed by Tukey's post hoc test (GraphPad


D

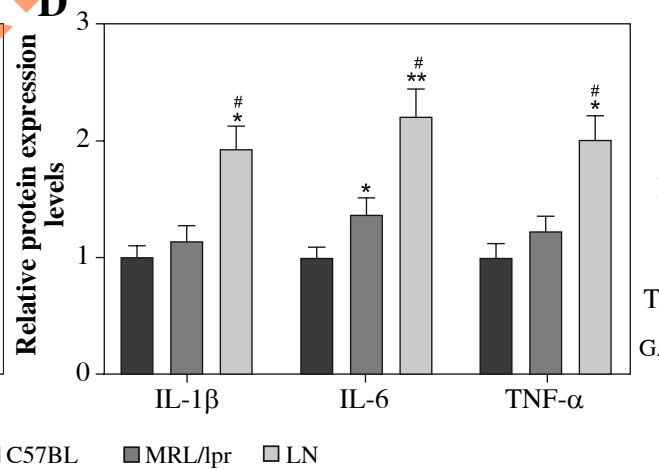

Prism; version 7; GraphPad Software, Inc.). $P<0.05$ was considered to indicate a statistically significant difference.

\section{Results}

\section{Lupus nephritis mice show high Tim-1 expression, strong autophagy and an inflammatory reaction}

RT-qPCR was performed to detect the expression of Tim-1 in LN mice. As shown in Figure 1A, the expression of Tim-1 was significantly upregulated in LN mice, compared with that in C57BL/6 or MRL ${ }^{\text {pr/pr }}$ lupus prone mice $(p<0.05)$. Meanwhile, the ratio of LC3B-II/LC3B-I in LN mice was much higher than that of $\mathrm{C} 57 \mathrm{BL} / 6$ or $\mathrm{MRL}^{\mathrm{Ipr} / \mathrm{pr}}$ lupus prone mice $(p<0.01$, Fig. 1B). A similar result was also observed in the expression of IL- $6, \mathrm{IL}-1 \beta$ and TNF- $\alpha$ at both mRNA and protein levels $(p<0.05, p<0.01$, $p<0.001$, Fig. 1C, D). These findings indicated that in-

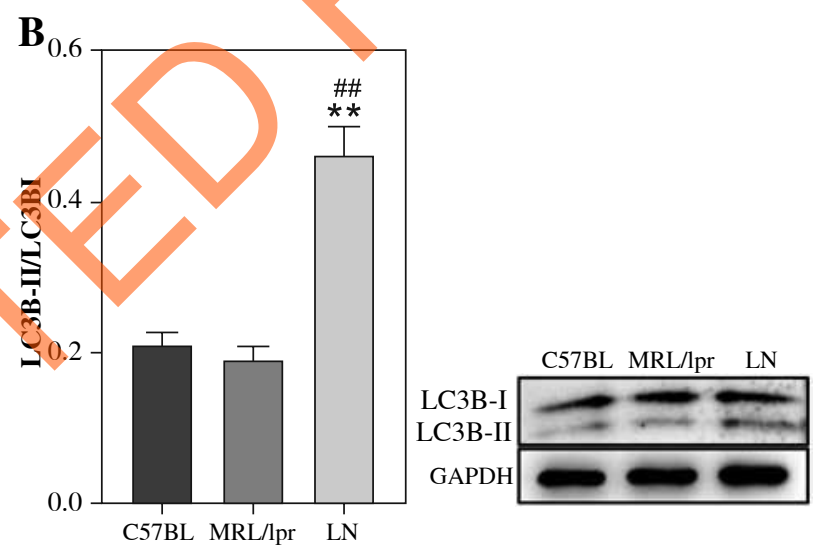

LN-lupus nephritis, Tim-1 - T cell immunoglobulin and mucin 1, GAPDH - glyceraldehyde-3-phosphate dehydrogenase, IL-6 - interleukin 6, TNF- $\alpha$-tumor necrosis factor $\alpha, I L-1 \beta$ - interleukin $1 \beta$, lpr-lymphoproliferation, RT-qPCR-real-time quantitative PCR

Fig. 1. LN mice show high Tim-1 expression, strong autophagy and an inflammatory reaction. A) Expression of Tim-1 in kidney tissues of mice was detected by western blot. The relative protein expression was quantified by normalizing to GAPDH. B) Expression of LC3B in kidney tissues of mice was detected by western blot. The ratio of LC3B-II/ LC3B-I was calculated by normalizing to GAPDH. C) Expression of IL-6, IL-1 $\beta$ and TNF- $\alpha$ in kidney tissues of mice was detected by RT-qPCR. D) Expression of IL-6, IL-1 $\beta$ and TNF- $\alpha$ in kidney tissues of mice was detected by western blot. Relative protein expression was quantified by normalizing to GAPDH. * $p<0.05$, ** $p<0.01$, *** $p<0.001 \mathrm{com}$ pared to control. ${ }^{\#} p<0.05,{ }^{\# \#} p<0.01$ compared to MRL/lpr 
flammatory responses were activated in LN and then induced autophagy. Taken together, the above data suggested that LN mice have increased Tim-1 expression, autophagy, and an inflammatory reaction.

\section{Tim-1 promotes autophagy and alleviates} inflammatory response in lupus nephritis cell model

Based on the increased Tim- 1 expression in LN mice, we speculated that Tim-1 was involved in the regulation of autophagy and the inflammatory response in the $\mathrm{LN}$ cell model. To verify this, we first determined the expression of Tim-1 based on RT-qPCR and western blot. As revealed in Figure 2A, the expression of Tim-1 was notably upregulated in IgG-treated podocytes at both mRNA and protein levels $(p<0.001)$, and Tim-1 overexpression significantly promoted this trend $(p<0.05)$. Compared with the control cells, no significant effect was found on the expression of autophagy-related proteins in Tim-1-transfected cells. However, compared with the IgG-treated cells, IgG plus Tim-1 resulted in enhancement of autophagy, as showed by the remarkably decreased p62 and the significantly decreased LC3BII/LC3B-I ratio ( $p<0.05$, Fig. 2B, C). Moreover, Tim- 1
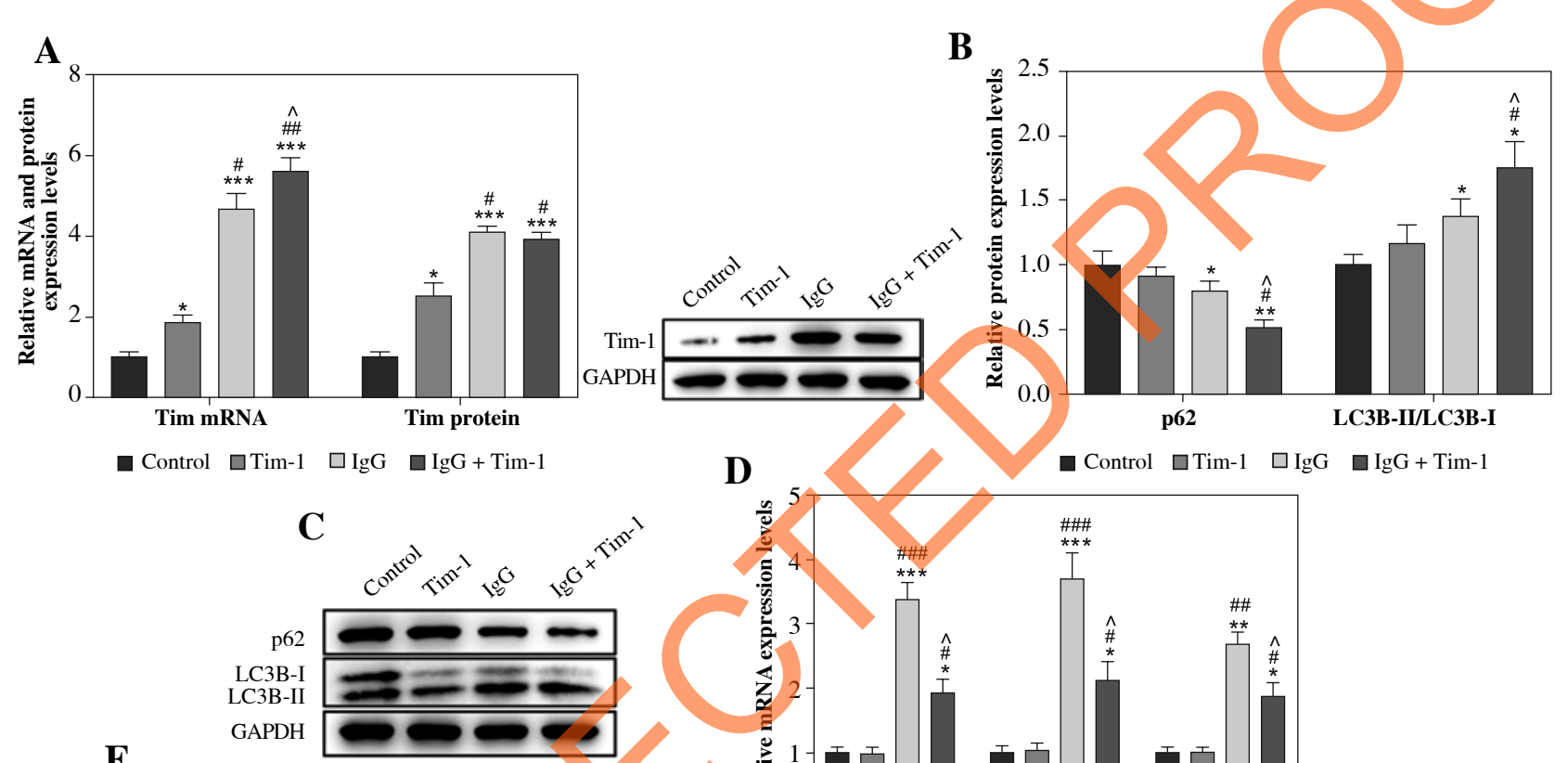

$\mathbf{E}$
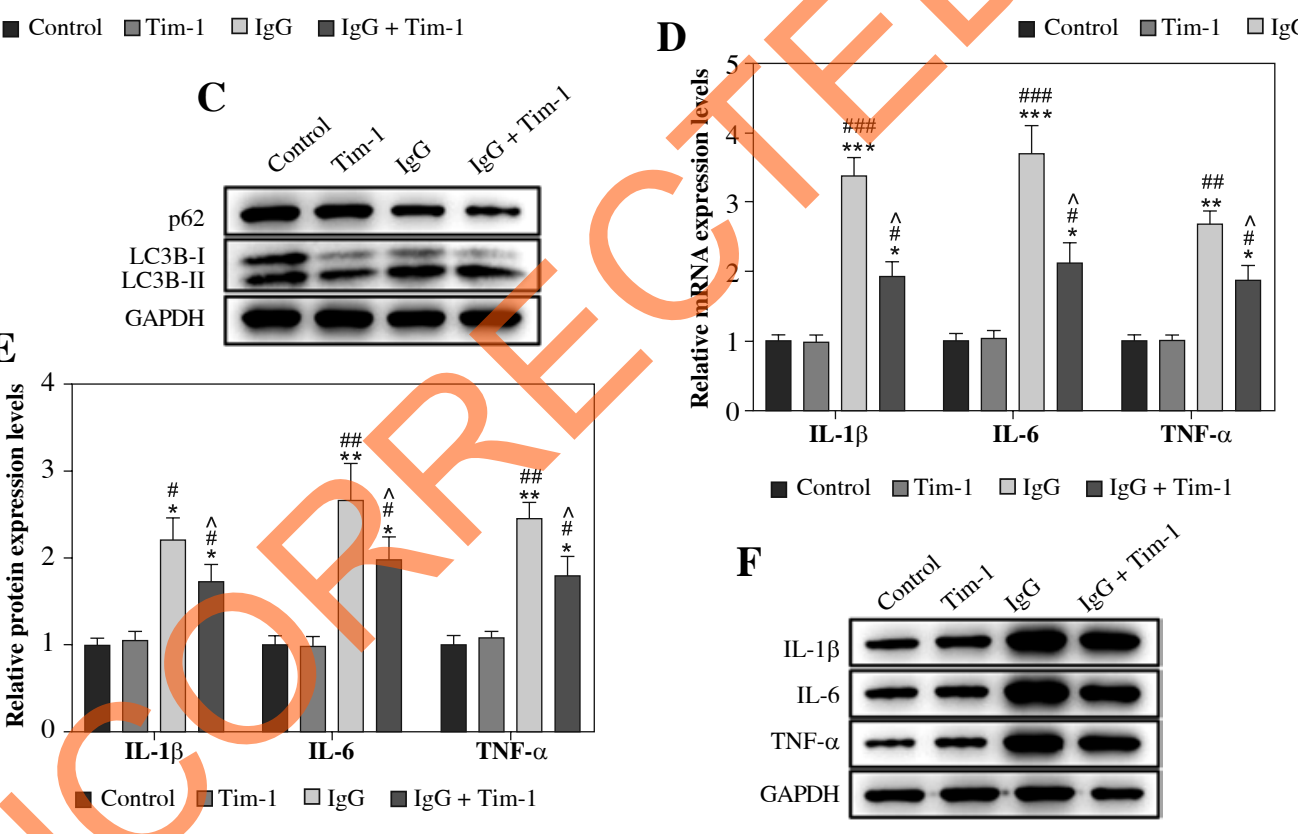

LN - lupus nephritis, Tim-1 - T cell immunoglobulin and mucin 1, GAPDH - glyceraldehyde-3-phosphate dehydrogenase, IL-6 - interleukin 6, $T N F-\alpha$-tumor necrosis factor $\alpha, I L-1 \beta$ - interleukin-1 $\beta$, lpr-lymphoproliferation, $R T$-qPCR - real-time quantitative PCR

Fig. 2. Tim-1 promotes autophagy and alleviates inflammatory response in LN cell model. Podocytes were treated with IgG, Tim-1 or Tim-1 + IgG. A) Expression of Tim-1 in podocytes was detected by RT-qPCR and western blot. Relative protein expression was quantified by normalizing to GAPDH. B, C) Protein expression of p62 and LC3B in podocytes was detected by western blot. The ratio of LC3B-II/LC3B-I and the relative expression of p62 were calculated by normalizing to GAPDH. D) Expression of IL-6, IL-1 $\beta$ and TNF- $\alpha$ in podocytes was detected by RT-qPCR. E, F) Protein expression of IL-6, IL-1 $\beta$ and TNF- $\alpha$ in podocytes was detected by western blot. Relative protein expression levels were quantified by normalizing to GAPDH. $* p<0.05, * * p<0.01, * * * p<0.001$ compared to control. ${ }^{*} p<0.05,{ }^{\# \#} p<0.05$, $\# p<0.05$ compared to Tim- $1 . \wedge p<0.05$ compared to IgG 
greatly alleviated IgG-induced increase of IL-6, IL-1 $\beta$ and TNF- $\alpha$ levels in podocytes at both mRNA and protein levels $(p<0.05$, Fig. 2D-F). Altogether, Tim-1 enhanced autophagy and alleviated inflammatory responses in IgG-induced podocytes.

\section{Tim-1 promotes proliferation and inhibits apoptosis in lupus nephritis cell model}

In order to detect the effect of Tim- 1 on the proliferation of LN cells, the CCK-8 assay was performed. As revealed in Figure $3 \mathrm{~A}$, much lower cell viability was observed in LN cells when compared to the control, and Tim-1 partially reversed this trend $(p<0.05)$. Meanwhile, limited apoptosis in podocytes was found after IgG treatment, while Tim-1 notably inhibited IgG-induced podocyte apoptosis ( $p<0.05$, Fig. 3B, C). To sum up, IgG-induced podocyte growth inhibition and apoptosis promotion can be significantly restored by Tim- 1 .

\section{Tim-1 alleviates the inflammatory response of lupus nephritis podocytes via inducing autophagy}

To further confirm the correlation between Tim-1 and autophagy in IgG-treated podocytes, we detected the effect of autophagy inhibitor (I) on Tim-1-mediated autophagy and inflammatory responses. As demonstrated in Figure 4A, B, the IgG-induced increase of Tim-1 expression was greatly enhanced by Tim- 1 overexpression, while autophagy inhibitor did not affect Tim-1 expression at either the mRNA or protein level $(p>0.05)$. However, the inhibitor obviously suppressed the Tim-1 induced enhancement of autophagy in the LN cell model, as shown in the inhibition of expression of $\mathrm{p} 62$ and the increase of LC3B-II/LC3B-I ratio $(p<0.05$, Fig. 4C, D). Similar, the inhibitory effect of Tim-1 on IgG-induced inflammatory responses in podocytes was also partially offset by autophagy inhibitor $(p<0.05$, Fig. 4E). In summary, Tim-1 significantly alleviated the inflammatory reaction of $\mathrm{LN}$ cells via inducing autophagy.

\section{Discussion}

Systemic lupus erythematosus is a prototypic autoimmune disease characterized by production of a diverse array of autoantibodies, complement activation, and deposition of immune complexes, causing tissue and organ damage [15, 24]. Many patients with SLE develop kidney disease, and LN is one of its most severe manifestations [14]. The immunologic features of LN contain autoantibodies directed against nuclear components and elevated pro-inflammatory cytokines, such as IL-1 $\beta$, IL-6, TNF- $\alpha$ and BAFF [25]. Clinical interventions

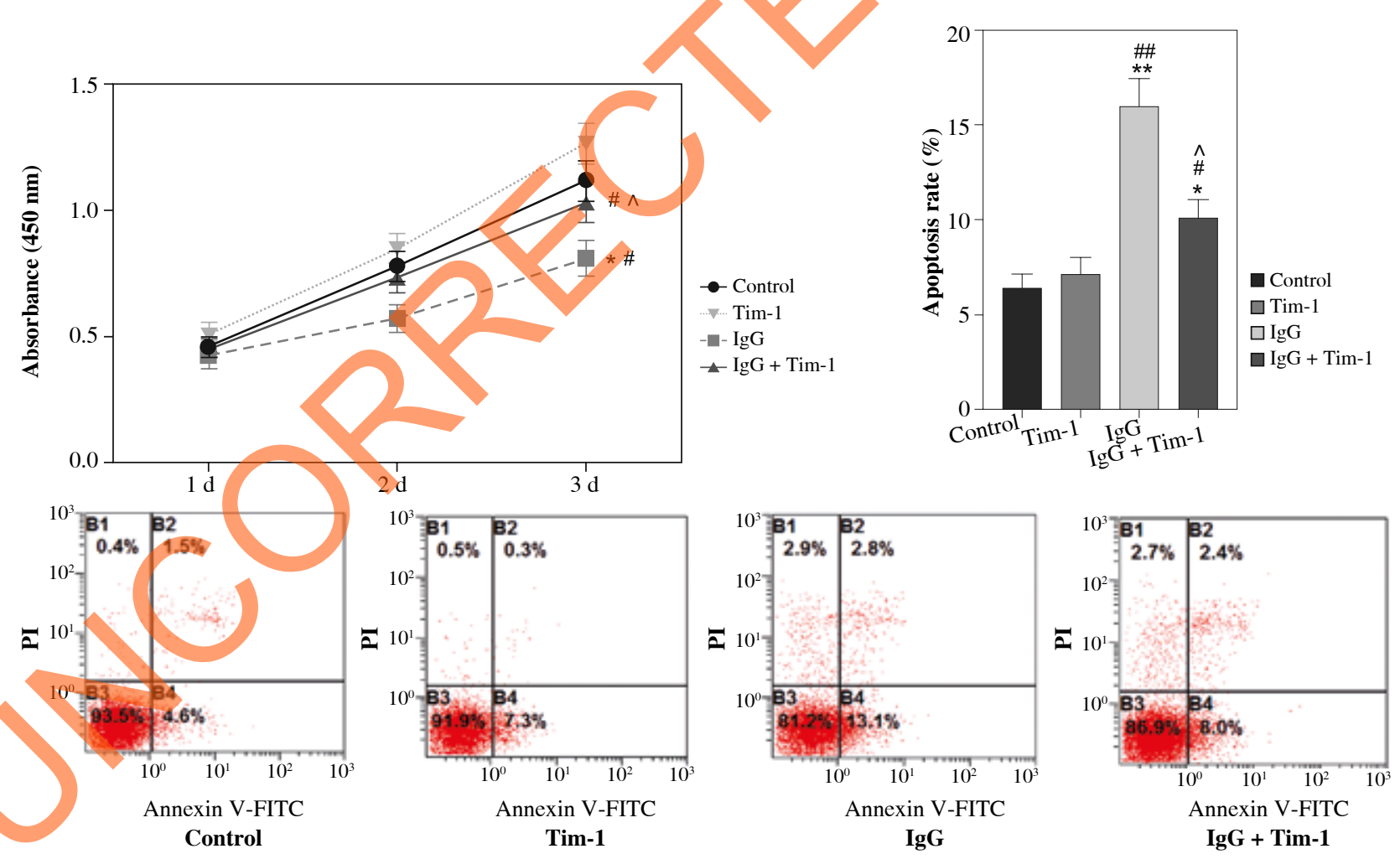

LN - lupus nephritis, CCK-8 - Cell Counting Kit-8, Tim-1 - T cell immunoglobulin and mucin 1

Fig. 3. Tim-1 promotes proliferation and inhibits apoptosis in LN cell model. A) After 24,48 or $72 \mathrm{~h}$ of incubation, viability of podocytes was tested by CCK- 8 assay. B, C) Apoptosis of podocytes was tested by flow cytometry. $* p<0.05$, ${ }^{* *} p<0.01$ compared to control. ${ }^{\#} p<0.05,{ }^{\#} p<0.01$ compared to Tim- $1 .{ }^{\wedge} p<0.05$ compared to IgG 


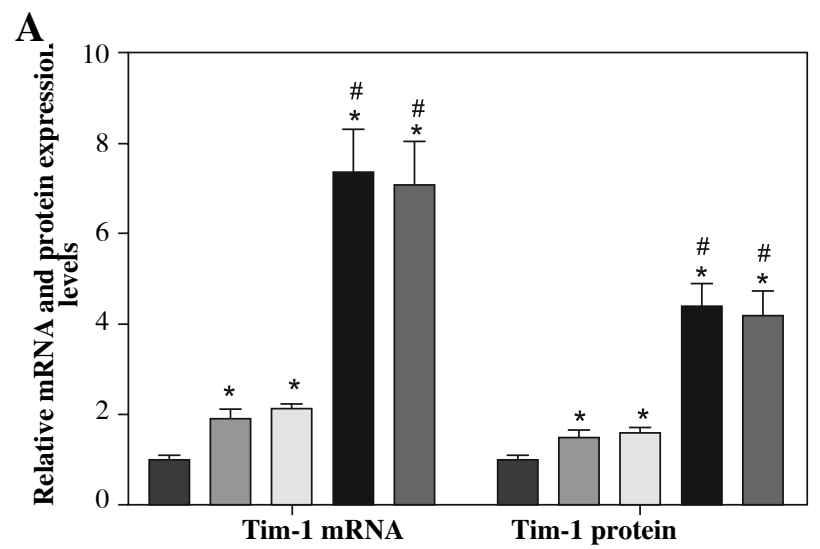

C
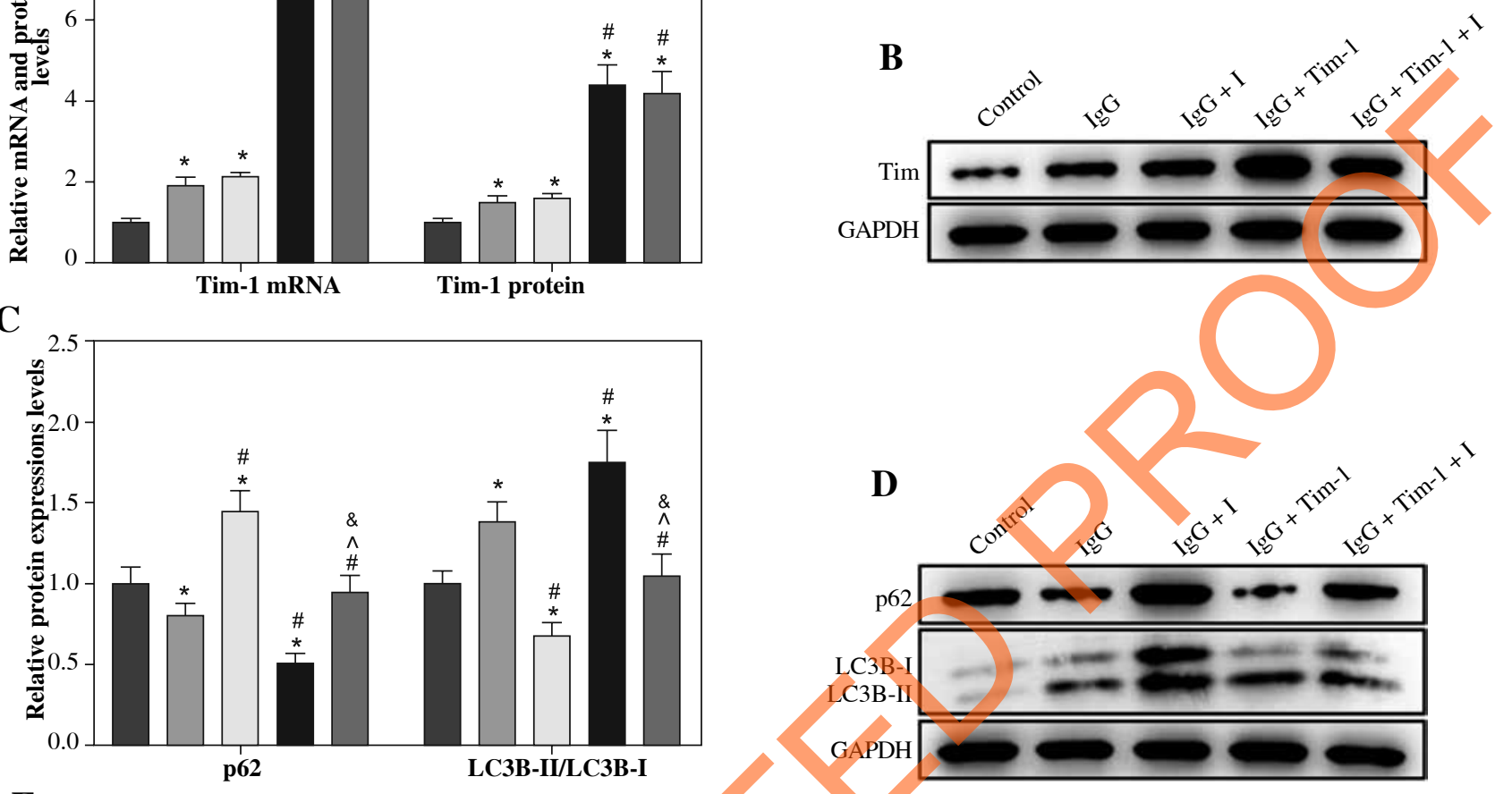

E
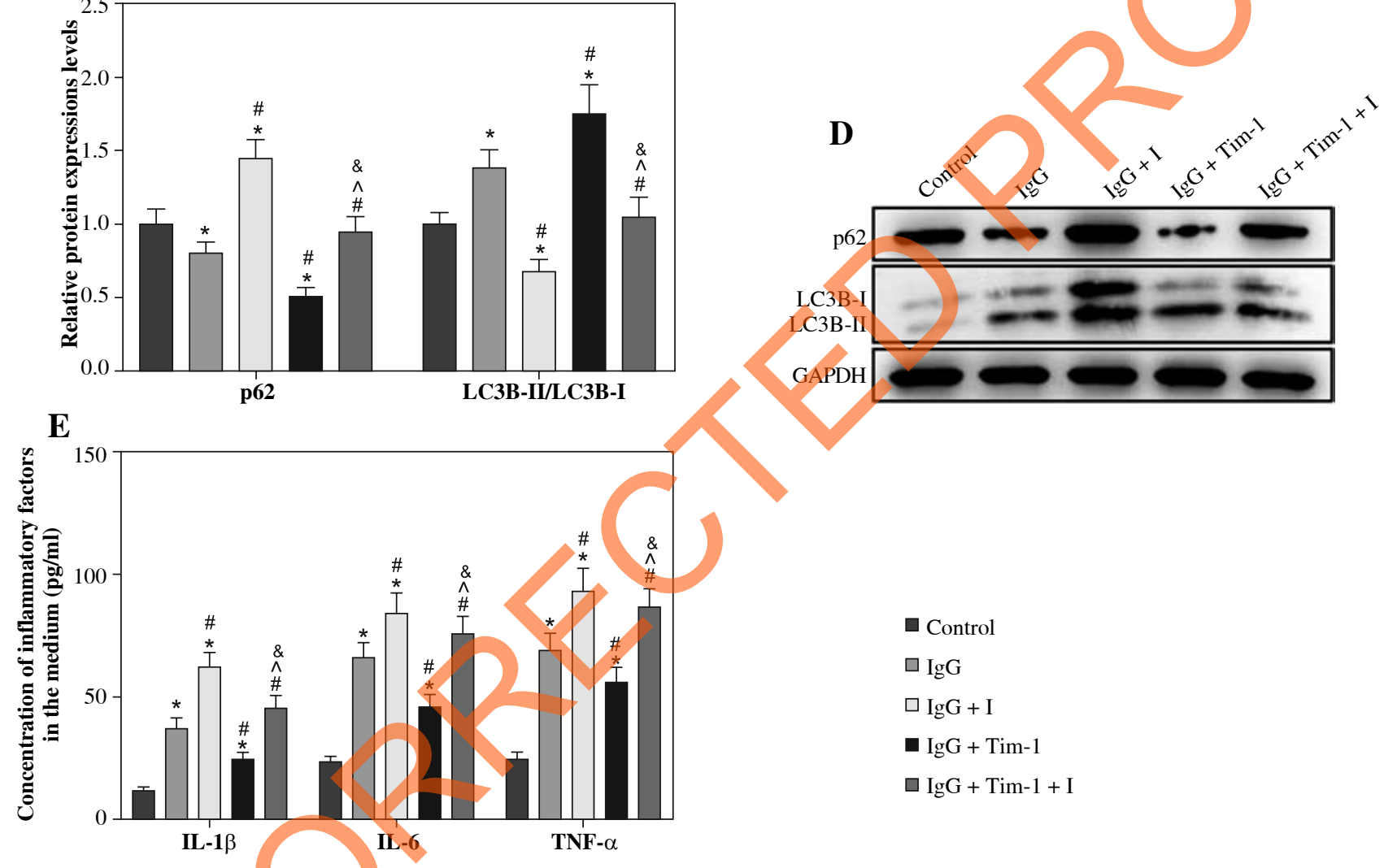

LN - lupus nephritis, Tim-1 - T cell immunoglobulin and mucin 1, GAPDH - glyceraldehyde-3-phosphate dehydrogenase, IL-6 - interleukin 6, TNF- $\alpha$-tumor necrosis factor $\alpha, I L-1 \beta$ - interleukin- $1 \beta$, lpr-lymphoproliferation, $R T$ - $q P C R$ - real-time quantitative PCR

Fig. 4. Tim-1 alleviates the inflammatory response of LN podocytes via inducing autophagy. Podocytes were treated with IgG, IgG + autophagy inhibitor (I), Tim-1 + IgG or Tim-1 + IgG + autophagy inhibitor (I). A) Expression of Tim-1 in podocytes was detected by RT-qPCR. B) Expression of Tim-1 in podocytes was detected by western blot. Relative protein expression was quantified by normalizing to GAPDH. C, D) Protein expression of LC3B and p62 in podocytes was detected by western blot. The ratio of LC3B-II/LC3B-I and the relative expression of p62 were calculated by normalizing to GAPDH. E) Levels of IL-6, IL- $1 \beta$ and TNF- $\alpha$ in supernatants of podocytes were detected by ELISA. * $p<0.05$ compared to control. ${ }^{\#} p<0.05$ compared to Tim- $1 .{ }^{\wedge} p<0.05$ compared to IgG + I. ${ }^{\star} p<0.05$ compared to IgG + Tim- 1

targeting multiple mediators such as IL-1 $\beta$, IL-6, TNF- $\alpha$ or BAFF have a very limited effect on LN [26], suggesting that other effector pathways may play a more dominant role in LN pathogenesis.
It has been reported that Tim-1 is involved in immune-related diseases. For example, Tim-1 can mediate $\mathrm{CD} 19^{+} \mathrm{CD} 24^{+} \mathrm{CD} 38^{+} \mathrm{B}$ cell function in primary biliary cholangitis [27]. Chiou et al. stated that Tim-1 plays a key 
role in the neuro-immune axis [28]. In addition, Tim-1 has been confirmed to be a mediator in immune function regulation [29]. Meanwhile, Tim-1 is known to participate in SLE and LN [15, 30]. In our study, Tim-1 was upregulated in LN, which was consistent with these previous reports. On the other hand, some studies indicated that only the $\mathrm{G}$ allele of Tim-1 at the $-1454 \mathrm{G} / \mathrm{A}$ site is a susceptibility variant associated with SLE [14, 31]. Based on these reports, it might be concluded that Tim-1 was downregulated in SLE due to the mutation of the -1454G/A site.

In addition, inflammatory responses and autophagy are involved in progression of $\mathrm{LN}[9,32]$. Wu et al. found that activation of autophagy may lead to the alleviation of LN severity [33]. Katsuyama et al. reported that immune responses are closely related to the development of $\mathrm{LN}$ [34]. Meanwhile, our data suggested that $\mathrm{LN}$ in mice is associated with autophagy and the inflammatory reaction. Thus, all these data suggested that Tim-1 alleviated the progression of $\mathrm{LN}$ via inhibiting immune responses and activating autophagy. Nevertheless, according to Zhou et al. autophagy could be negatively associated with podocyte injury during the progression of lupus nephritis [35]. Our research was similar to Zhou et al., suggesting that autophagy might inhibit lupus nephritis progression.

To further verify the correlation among Tim-1, autophagy and the immune reaction, podocytes were treated with $\mathrm{IgG}$ to mimic $\mathrm{LN}$ in vitro. The results showed that Tim-1 could inhibit the expression of IL-6, TNF- $\alpha$, IL- $1 \beta$, and LC3II/LC3I ratio and increase the p62 level in IgG-treated podocytes. Consistently, previous reports confirmed the role of immune responses and autophagy in LN [36, 37]. Thus, it could be suggested that Tim-1 could act as an inhibitor in immune responses, as well as a promoter in autophagy.

Based on the above data, we sought to further explore the effect of Tim-1 on growth of IgG-treated podocytes. Thus, flow cytometry and the CCK -8 assay were performed. Our findings demonstrated that Tim-1 could reverse IgG-induced podocyte growth inhibition. In contrast, Zheng et al. found that Tim-1 knockdown could inhibit the apoptosis of neurons in cerebral ischemia-reperfusion injury [38]. The discrepancy might due to different type of diseases.

To confirm the correlation between Tim-1 and autophagy in $\mathrm{LN}$, podocytes were treated with an autophagy inhibitor. The data revealed that the autophagy inhibitor could reverse Tim-1-induced activation of autophagy in LN. Autophagy has been regarded as an evolutionarily process by which eukaryotic cells maintain intracellular homeostasis and adapt themselves to cellular stress to survive. The increase of autophagy activities often leads to inhibition of apoptosis [39]. Consistent with this background, our finding suggested that Tim-1 inhibited the apoptosis of IgG-treated podocytes via inducing autophagy. P62 and LC3B-II/LC3B-I ratio are key factors in activation of cell autophagy [40, 41]. Downregulation of p62 and increase of LC3B-II/LC3B-I could lead to the occurrence of autophagy $[42,43]$. In addition, Chu et al. stated that Tim-1 could trigger autophagy in dengue virus infection [22]. Our research was in line with these previous reports, suggesting that Tim-1 could act as a promoter in LN. Meanwhile, Brooks et al. found that Tim-1-mediated T-cell proliferation was closely associated with cell autophagy [23]. Since $\mathrm{LN}$ is confirmed to be a type of auto-immune disease, the correlation between T-cell-mediated immune responses and autophagy in LN needs to be investigated in the future.

There are some limitations in this research. For example, some checkpoints for T cell-mediated cytokines need to be detected in LN. In addition, the correlation between Tim-1 and autophagy remains to be further explored. Hence, more investigations are needed in the future.

\section{Conclusions}

In conclusion, Tim-1 significantly inhibited the progression of $\mathrm{LN}$ via inducing autophagy, which might serve as a potential new target for the treatment of LN.

\section{Ethics approval}

All animals were kept in a pathogen-free environment and fed ad lib. The procedures for care and use of animals were approved by the Ethics Committee of the General Hospital of Ningxia Medical University and all applicable institutional and governmental regulations concerning the ethical use of animals were followed.

\section{Availability of data and material}

The datasets used or analyzed during the current study are available from the corresponding author on reasonable request.

\section{Funding}

This study was supported by a Science and Technology project of Ningxia Hui Autonomous Region (Grant No.: 2011056).

The authors declare no conflict of interest.

\section{References}

1. Varley JA, Andersson M, Grant E, et al. (2020): Absence of neuronal autoantibodies in neuropsychiatric systemic lupus erythematosus. Ann Neurol 88: 1244-1250.

2. Aringer M, Costenbader K, Daikh D, et al. (2019): 2019 European League Against Rheumatism/American College of Rheumatology classification criteria for systemic lupus erythematosus. Ann Rheum Dis 78: 1151-1159. 
3. Kayacan Erdogan E, Gokcen N, Badak SO, et al. (2021): Sacroiliitis in systemic lupus erythematosus: the rates of involvement of the forgotten joint. Z Rheumatol 80: 447-455.

4. Bajema IM, Wilhelmus S, Alpers CE, et al. (2018): Revision of the International Society of Nephrology/Renal Pathology Society classification for lupus nephritis: clarification of definitions, and modified National Institutes of Health activity and chronicity indices. Kidney Int 93: 789-796.

5. Ramanujam M, Steffgen J, Visvanathan S, et al. (2020): Phoenix from the flames: Rediscovering the role of the CD40CD40L pathway in systemic lupus erythematosus and lupus nephritis. Autoimmun Rev 19: 102668.

6. Ward M, Tektonidou MG (2020): Belimumab as add-on therapy in lupus nephritis. N Engl J Med 383: 1184-1185.

7. Fouda ME, Nour El Din DM, Mahgoub MY, et al. (2020): Genetic variants of microRNA-146a gene: an indicator of systemic lupus erythematosus susceptibility, lupus nephritis, and disease activity. Mol Biol Rep 47: 7459-7466.

8. Truszewska A, Wirkowska A, Gala K, et al. (2020): Cellfree DNA profiling in patients with lupus nephritis. Lupus 29: 1759-1772.

9. Lou H, Wojciak-Stothard B, Ruseva MM, et al. (2020): Autoantibody-dependent amplification of inflammation in SLE. Cell Death Dis 11: 729.

10. Kong X, Fu M, Niu X, Jiang H (2020): Comprehensive analysis of the expression, relationship to immune infiltration and prognosis of TIM-1 in cancer. Front Oncol 10: 1086.

11. Zhou P, Fei M, Han Y, et al. (2020): Knockdown of T cell immunoglobulin and mucin 1 (Tim-1) suppresses glioma progression through inhibition of the cytokine-PI3K/AKT pathway. Onco Targets Ther 13: 7433-7445.

12. Xu Y, Wu K, Han S, et al. (2020): Astilbin combined with lipopolysaccharide induces IL-10-producing regulatory B cells via the STAT3 signalling pathway. Biomed Pharmacother 129: 110450.

13. Li J, Qiu Y, Li L, et al. (2020): Histone methylation inhibitor DZNep ameliorated the renal ischemia-reperfusion injury via inhibiting TIM-1 mediated T cell activation. Front Med (Lausanne) 7: 305 .

14. Li WX, Chen GM, Yuan H, et al. (2011): Polymorphisms of the TIM-1 and TIM-3 genes are not associated with systemic lupus erythematosus in a Chinese population. Mutagenesis 26: 507-511.

15. Nozaki Y, Kitching AR, Akiba H, et al. (2014): Endogenous Tim-1 promotes severe systemic autoimmunity and renal disease MRL-Fas(lpr) mice. Am J Physiol Renal Physiol 306: F1210-1221.

16. Xie Q, Liu Y, Li X (2020): The interaction mechanism between autophagy and apoptosis in colon cancer. Transl Oncol 13: 100871 .

17. Chen X, Sun K, Zhao S, et al. (2020): Irisin promotes osteogenic differentiation of bone marrow mesenchymal stem cells by activating autophagy via the $\mathrm{Wnt} /$ /beta-catenin signal pathway. Cytokine 136: 155292.

18. Sarkar A, Gandhi V (2021): Activation of ATM kinase by ROS generated during ionophore-induced mitophagy in human T and B cell malignancies. Mol Cell Biochem 476: 417-423.

19. Chen G, Jin X, Luo D, et al. (2020): $\beta$-Adrenoceptor regulates contraction and inflammatory cytokine expression of human bladder smooth muscle cells via autophagy under pathological hydrostatic pressure. Neurourol Urodyn 39: 2128-2138.
20. Yang F, He Y, Zhai Z, Sun E (2029): Programmed cell death pathways in the pathogenesis of systemic lupus erythematosus. J Immunol Res 2019: 3638562.

21. Yang SR, Hsu WH, Wu CY, et al. (2020): Accelerated, severe lupus nephritis benefits from treatment with honokiol by immunoregulation and differentially regulating NF-kappaB/ NLRP3 inflammasome and sirtuin 1/autophagy axis. FASEB J 34: 13284-13299.

22. Chu LW, Yang CJ, Peng KJ, et al. (2019): TIM-1 as a signal receptor triggers Dengue virus-induced autophagy. Int J Mol Sci 20: 4893.

23. Brooks CR, Yeung MY, Brooks YS, et al. (2015): KIM-1TIM-1-mediated phagocytosis links ATG5-/ULK1-dependent clearance of apoptotic cells to antigen presentation. EMBO J 34: 2441-2464.

24. Cai XZ, Huang WY, Qiao Y, et al. (2015): Downregulation of TIM-3 mRNA expression in peripheral blood mononuclear cells from patients with systemic lupus erythematosus. Braz J Med Biol Res 48: 77-82.

25. Li MZ, Lai DH, Zhao HB, et al. (2020): SOCS3 overexpression enhances ADM resistance in bladder cancer T24 cells. Eur Rev Med Pharmacol Sci 24: 11985.

26. Colucci M, Ruggiero B, Gianviti A, et al. (2021): IgM on the surface of T cells: a novel biomarker of pediatric-onset systemic lupus erythematosus. Pediatr Nephrol 36: 909-916.

27. Chen Q, Lai L, Chi X, et al. (2020): CD19(+)CD24(hi) CD38(hi) B cell dysfunction in primary biliary cholangitis. Mediators Inflamm 2020: 3019378.

28. Chiou B, Lucassen E, Sather M, et al. (2018): Semaphorin4A and H-ferritin utilize Tim-1 on human oligodendrocytes: A novel neuro-immune axis. Glia 66: 1317-1330.

29. Du P, Xiong R, Li X, Jiang J (2016): Immune regulation and antitumor effect of TIM-1. J Immunol Res 2016: 8605134.

30. Yuan H, Yao YS, Chen GM, et al. (2016): Decreased serum levels of T-cell immunoglobulin mucin-1 and T-cell immunoglobulin mucin-3 in systemic lupus erythematosus patients. J Biol Regul Homeost Agents 30: 123-129.

31. Yu Y, Zhu C, Zhou S, Chi S (2018): Association between C1q, TRAIL, and Tim-1 gene polymorphisms and systemic lupus erythematosus. Genet Test Mol Biomarkers 22: 546553.

32. Dong Y, Zhang Z, Liu H, et al. (2020): Exacerbating lupus nephritis following BPA exposure is associated with abnormal autophagy in MRL/lpr mice. Am J Transl Res 12: 649-659.

33. Wu CY, Hua KF, Chu CL, et al. (2020): Tris DBA ameliorates accelerated and severe lupus nephritis in mice by activating regulatory $\mathrm{T}$ cells and autophagy and inhibiting the NLRP3 inflammasome. J Immunol 204: 1448-1461.

34. Katsuyama T, Li H, Krishfield SM, et al. (2021): Splicing factor SRSF1 limits IFN-gamma production via RhoH and ameliorates experimental nephritis. Rheumatology (Oxford) 60: 420-429.

35. Zhou XJ, Klionsky DJ, Zhang H (2019): Podocytes and autophagy: a potential therapeutic target in lupus nephritis. Autophagy 15: 908-912.

36. Zhao Y, Wei W, Liu ML (2020): Extracellular vesicles and lupus nephritis - New insights into pathophysiology and clinical implications. J Autoimmun 115: 102540.

37. Kanno Y, Miyashita M, Seishima M, Matsuo O (2020): alpha2AP is associated with the development of lupus nephritis through the regulation of plasmin inhibition and inflammatory responses. Immun Inflamm Dis 8: 267-278. 
38. Zheng Y, Wang L, Chen M, et al. (2019): Inhibition of T cell immunoglobulin and mucin-1 (TIM-1) protects against cerebral ischemia-reperfusion injury. Cell Commun Signal 17: 103.

39. Beckerman P, Bi-Karchin J, Park AS, et al. (2017): Transgenic expression of human APOL1 risk variants in podocytes induces kidney disease in mice. Nat Med 23: 429-438.

40. Xie Y, Chen L, Zhou J, et al. (2020): TGFbeta signaling-induced miRNA participates in autophagic regulation by targeting PRAS40 in mesenchymal subtype of glioblastoma. Cancer Biol Med 17: 664-675.

41. Silva KAS, Leary EV, Olver TD, et al. (2020): Tissue-specific small heat shock protein 20 activation is not associated with traditional autophagy markers in Ossabaw swine with cardio-metabolic heart failure. Am J Physiol Heart Circ Physiol 319: H1036-H1043.

42. Wang L, Liu H, He Q, et al. (2020): Galangin ameliorated pulmonary fibrosis in vivo and in vitro by regulating epithelialmesenchymal transition. Bioorg Med Chem 28: 115663.

43. Huang SC, Adhikari S, Brownell JE, et al. (2020): Discovery and optimization of pyrazolopyrimidine sulfamates as ATG7 inhibitors. Bioorg Med Chem 28: 115681. 